\title{
Poorly differentiated and anaplastic thyroid carcinomas: chromosomal and oligo-array profile of five new cell lines
}

\author{
RF Rodrigues', L Roque*,', T Krug ${ }^{2}$ and $\mathbf{V}$ Leite $^{3}$ \\ 'Cytogenetic Laboratory, Centro de Immunologia e Patologia Molecular, Portuguese Cancer Institute, R. Professor Lima Basto, Lisbon, Portugal; ${ }^{2}$ Gene \\ Express, Lda, Taguspark, Oeiras, Portugal; ${ }^{3}$ Valeriano Leite: Molecular Endocrinology Laboratory, Centro de Immunologia e Patologia Molecular, \\ Portuguese Cancer Institute, R. Professor Lima Basto, Lisbon, Portugal
}

Information on gene alterations associated to poorly differentiated (PDTC) and anaplastic thyroid carcinomas (ATC) is scarce. Using human cancer cell lines as a tool for gene discovery, we performed a cytogenetic and oligo-array analysis in five new cell lines derived from two PDTC and three ATC. In PDTC we evidenced, as important, the involvement of the MAPK/ERK kinase pathway, and downregulation of a group of suppressor genes that include E-cadherin. In ATC, downregulation of a specific group of oncosuppressor genes was also observed. Our ATC cell lines presented chromosomal markers of gene amplification, and we were able to identify for the first time the nature of the involved amplicon target genes. We found that the main molecular differences between the two cell line types were related to signal transduction pathways, cell adhesion and motility process. TaqMan experiments performed for five amplicon target genes and for two genes, which allowed a clear distinction between ATC and PDTC: CDHI 3 and PLAU corroborated array results, not only in the cell lines, but also in an additional set of primary I4 PDTC and three ATC. We suggest that our findings may represent new tools for the development of more effective therapies to the hitherto untreatable ATC.

British Journal of Cancer (2007) 96, 1237-1245. doi:10.1038/sj.bjc.6603578 www.bjcancer.com

Published online 3 April 2007

(C) 2007 Cancer Research UK

Keywords: thyroid; $\mathrm{CGH}$; expression; profile

Thyroid malignant neoplasms of follicular cell origin are classified into four main categories: the differentiated papillary (PTC) and follicular (FTC) carcinomas, the poorly differentiated (PDTC) and the undifferentiated or anaplastic (ATC) carcinomas. The differentiated forms are the most common. Poorly differentiated thyroid carcinomas and ATC account for only $5-10 \%$ of the follicular malignancies; however, although rare, they represent a major challenge in oncology as they have a high morbidity and mortality rate (DeLelllis and Williams, 2004).

In recent years some progress has been obtained in the identification of the genomic changes associated to the pathogenesis of DTC. In PTC, it is now clear that, in a substantially fraction of tumours, malignant transformation takes place through the constitutive activation of effectors along the MAPK/ERK kinase pathway (Mellilo et al, 2005), and in FTC, the PAX8/PPRA $\gamma$ fusion gene was determined to be an important event in the development of a subset of these carcinomas (Lacroix et al, 2005). At variance, the genetic information concerning PDTC and ATC is very limited. Only 27 PDTC have been evaluated by comparative genomic hybridisation (CGH) analysis, and as far as we are aware, there are no microarray studies on this entity (Wreesmann et al, 2002; Rodrigues et al, 2004). In ATC, it was demonstrated that TP53 (Wynford-Thomas, 1997) and $\beta$-catenin (Garcia-Rostan et al,

\footnotetext{
*Correspondence: Dr L Roque;

E-mail: Iroque@ipolisboa.min-saude.pt

Received 27 April 2006; revised 21 November 2006; accepted II December 2006; published online 3 April 2007
}

1999) mutations as well as the overexpression of OEATC1 (Mizutani et al, 2005) and AURKB (Sorrentino et al, 2005) were associated with ATC phenotype. These genes were demonstrated to be key effectors in anaplastic transformation, being responsible for evasion to apoptosis, enhanced tumour growth and replicative potential. However, although all efforts made, the molecular events related to their high invasion capacity, sustained angiogenesis and therapeutic resistance remain to be identified. By chromosomal studies it has become clear that these neoplasms were frequently characterised by cytogenetic markers of gene amplification: double minutes $(\mathrm{dm})$ and homogeneously staining regions (hsr) (Roque et $a l, 1998)$. In other tumour types, such as neuroblastomas, the evaluation of these markers allowed the identification of specific oncogenes as targets of the amplification process, being found to be associated with a more aggressive biologic behaviour (Brodeur, 2003). In ATC, the identification of the amplified target genes has not been accomplished yet.

Cancer cell lines represent a pool of cells with an unlimited renewal and proliferative potential, and have proven to be invaluable in the research of aggressive rare tumours and a fundamental tool for gene discovery. The number of cell lines derived from poorly differentiated and ATC is small with just two PDTC cell lines (NPA and SMP) being referred in the literature (Asakawa et al, 1996; Ringel et al, 2000; Onda et al, 2004).

In our laboratory we were able to establish cell lines from two PDTC and three ATC, which we evaluated by conventional cytogenetics, CGH and oligonucleotide array analysis. It was our aim to characterise the cell lines at chromosomal and expression levels, providing information on the yet unknown deregulated 
genetic mechanisms associated to these two forms of aggressive thyroid neoplasias, namely to identify the target genes located at the amplicon units of the ATC cell lines.

From our results, it was evidenced that both cancer cell line types were characterised by largely altered chromosomal and expression profiles. In PDTC, we noted as significant the deregulation of MAPK pathway effectors and of a group of tumour suppressor genes. In ATC, we identified 40 genes as amplicon targets. Comparison of array results between the two types of cell lines revealed significant differences in the expression levels of genes related to signal transduction, cell adhesion, and motility. Array data were validated at RNA level through the performance of quantitative reverse transcription-polymerase chain reaction (RT-PCR) experiments for ARNTL2, AURKA, CDH13, CLDN1, $P L A U, P M A I P 1$, and RBAK.

\section{MATERIALS AND METHODS}

\section{Tumour specimens, conventional cytogenetics and CGH analysis}

Tissue samples were obtained by thyroidectomy from two patients with poorly differentiated papillary carcinomas and three patients with anaplastic carcinomas. Tumours were classified according to the criteria described in the Armed Forces Institute of Pathology (Rosai et al, 1992) and revaluated according to WHO histological classification of thyroid and parathyroid tumours by DeLellis and Williams (2004). Representative haematoxylin-eosin tissue sections from each of the primary tumours are shown in Figure 1A-F. A summary of the patient's clinical and histological data and the cell lines designation is depicted in Table 1 .

Tumour cells were grown in Rosewell Park Memorial Institute 1640 (RPMI 1640) medium (Gibco-Invitrogen, Scotland, UK), containing $10 \%$ fetal calf serum (FCS) (Gibco-Invitrogen, Scotland, $\mathrm{UK}$ ) and $1 \%$ antibiotic/antimicotic solution (Sigma-Aldrich Chemie, GmbH, Germany).

For conventional cytogenetic analysis cells were harvested after a 3- to 5-h treatment with colchicine (Karyomax-Gibco-Invitrogen, Scotland, UK). The metaphases obtained were GTG banded and karyotypes described according to International System for Human Cytogenetic Nomenclature (ISCN) (1995) (Mitelman, 1995).

Comparative genomic hybridisation of the five cell lines and evaluation of the obtained results were performed as described previously (Rodrigues et al, 2004).
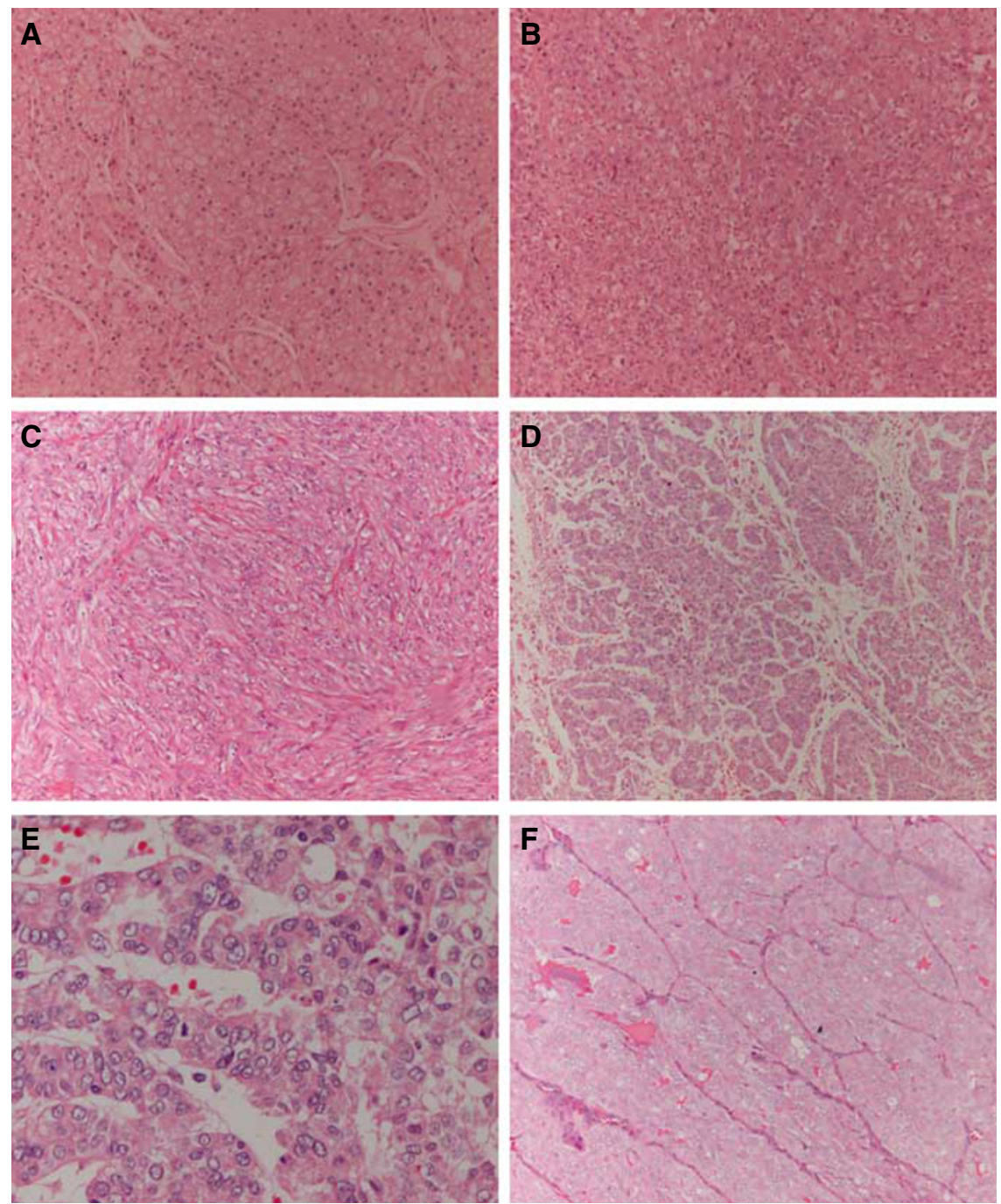

Figure I Representative haematoxylin-eosin-stained tissue sections of the primary poorly differentiated and anaplastic thyroid carcinomas from which the cell lines were derived. (A) T235 (original amplification 100); (B) T238 (original amplification I00); (C) T24I (original amplification I00); (D) T243 (original amplification 100); (E) T243 (original amplification 400); (F) T35I (original amplification I00). 
Table I Clinical and histological data of the five cancer patients

\begin{tabular}{|c|c|c|c|c|}
\hline Case & Sex/age & $\begin{array}{l}\text { Date of } \\
\text { surgery }\end{array}$ & Deceased & Histologic diagnosis \\
\hline T235 & $\mathrm{F} / 77$ & | 2.05.97 & | 7.06 .97 & $\begin{array}{l}\text { Anaplastic thyroid } \\
\text { carcinoma }\end{array}$ \\
\hline T238 & $\mathrm{F} / 74$ & 20.05 .97 & 1.06 .97 & $\begin{array}{l}\text { Anaplastic thyroid } \\
\text { carcinoma }\end{array}$ \\
\hline T24I & $\mathrm{F} / 72$ & 30.06 .97 & 30.11 .97 & $\begin{array}{l}\text { Anaplastic thyroid } \\
\text { carcinoma }\end{array}$ \\
\hline T243 & $M / 67$ & 20.07 .97 & - & $\begin{array}{l}\text { Poorly differentiated } \\
\text { papillary thyroid carcinoma }\end{array}$ \\
\hline T35। & $M / 58$ & 10.01 .00 & 20.10 .02 & $\begin{array}{l}\text { Poorly differentiated } \\
\text { papillary thyroid carcinoma }\end{array}$ \\
\hline
\end{tabular}

\section{Oligonucleotide microarray analysis}

Human thyroid total RNA from Clontech ${ }^{\circledR}$ (consisting in a pool of thyroid RNA obtained from 65 individuals who died from sudden death) was used as a normal baseline reference for microarray experiments. Total RNA from the cell lines was extracted using Trizol $^{\circledR}$ (Gibco-Invitrogen, Scotland, UK), and cleaned up using Quiagen columns according to manufacturer's protocols. RNA integrity was assessed in the Bioanalyser Agilent 2100 and by visualisation of 28 and 18-s bands in agarose gel electrophoresis. Ribonucleic acid concentration was determined by spectrophotometry. For hybridisation the commercial GeneChip ${ }^{\circledR}$ Human Genome U133 Plus 2.0 Array from Affymetrix ${ }^{\mathbb{R}}$ were used.

For each cell line a pool of RNA derived from different passages was obtained, and samples were processed according to the experimental procedures specified by Affymetrix ${ }^{\mathbb{R}}$.

The obtained microarrays were scanned in GeneChip ${ }^{\circledR}$ Scanner 3000 from Affymetrix ${ }^{\mathbb{R}}$, controlled by a workstation with the GeneChip ${ }^{\mathbb{B}}$ Operating Software (GCOS) version 1.1.

\section{Statistical analysis}

The software chosen for the analysis was the DNA-Chip Analyser (dChip) (copyright 2000-2004 Wang Lab, Harvard School of Public Health and Dana-Farber Cancer Institute). In the first step of the statistical analysis the arrays were normalised by the invariant set normalisation method, so that all non-biological variables were reduced, followed by a model-based expression analysis, using the model PM-only. The genes were filtered, so that those absent in all the samples were eliminated from the analysis. We considered genes to be differentially expressed between samples, when the lower bound of fold change (LBFC) was at least 2 , with a confidence of $90 \%$. In order to determine relevant genes overexpressed in the high-level amplification regions (HLA), we chose the set of genes that were found to have (i) an LBFC $>5$ in the comparison of the cell with the HLA to normal thyroid, within the HLA chromosomal region, and (ii) were commonly differentially expressed in the comparison of the three ATC cell lines to normal thyroid. The obtained data were then subject to hierarchical clustering and principal components analysis (PCA). The genes were also classified according to 'gene ontology' and 'chromosome location' using classify genes tool of D-Chip. We regarded only the $P$ significant genes $(P<0.001)$ for this classification.

\section{Quantitative real-time RT-PCR}

To validate the array results, quantitative real-time RT-PCR was performed on seven genes: Aryl Hydrocarbon Receptor Nuclear Translocator-like 2 (ARNTL2); Aurora Kinase A (AURKA), Cadherin 13 ( $C D H 13)$, Claudin 1 (CLDN1), phorbol-12-myristate- 13-acetate-induced protein 1 (PMAIP1), plasminogen activator urokinase $(P L A U)$, and $\mathrm{RB}$-associated $\mathrm{KRAB}$ repressor $(R B A K)$ using an ABI PRISM 7900HT Sequence Detection System (Applied Biosystems, Foster City, CA, USA). Sequence-specific primers and probes were selected from the Assay-on-Demand products (Applied Biosystems). As endogenous control we selected the housekeeping gene glyceraldehyde-3-phosphate dehydrogenase (GAPDH) from Applied Biosystems (HS:99999905_m1). The conditions of the TaqMan ${ }^{\mathbb{R}}$ PCR were as follows: $95^{\circ} \mathrm{C}$ for $10 \mathrm{~min}$, followed by $40-45$ cycles of $95^{\circ} \mathrm{C}$ for $15 \mathrm{~s}$ and $60^{\circ} \mathrm{C}$ for $1 \mathrm{~min}$. The relative expression of each sample was calculated with respect to a standard calibration curve that represents a serial dilution of cDNA positive for the expression of the gene in analysis. Each sample was analysed three times and each PCR experiment included at least one non-template control well.

ARNTL2, AURKA, CLDN1, PMAIP1, and RBAK were chosen as representative overexpressed genes at the HLA regions. TaqMan ${ }^{\mathbb{R}}$ PCR experiments were performed in the three ATC cell lines reported herein as well as in three ATC independent primary tumours.

CDH13 and PLAU were chosen because they were genes that accordingly to array data allowed a clear distinction between ATC and PDTC. Experiments were performed in the five cell lines described herein, in 14 PDTC primary tumours and in the previously aforementioned three ATC additional samples.

\section{RESULTS}

\section{Conventional cytogenetic and CGH analysis}

Tumours were characterised by karyotypes with both structural and numerical abnormalities (chromosomal supplementary data). Several populations were found in all cell lines. Different populations presented roughly the same alterations, differing only in ploidy. Maintenance of the karyotypes was observed for all cell lines.

By CGH all cell lines presented multiple chromosomal imbalances. No significant modifications were observed throughout time at CGH level in any of the carcinomas after cell line establishment. A summary of the CGH imbalances is depicted in Figure 2A. Overall, gains of DNA sequences were more frequent than losses. High-level amplification regions were observed in the following cell lines: in T235 at regions 3q24-qter, 7q11.2-q22, 12pter-p11 and chromosome 20; in T238 at 18q21; and in T241 at 5pter-p12, 7pter-p21, 14q10-qter, and 20p11.2-qter (Figure 2B-D).

Comparative genomic hybridisation of the corresponding primary tumours have been performed and reported previously (Rodrigues et al, 2004). The chromosomal gains and losses observed for each of the cell lines and respective primary tumours were represented in an excel table (CGH supplementary data). Analysis of these data allowed to verify that although CGH alterations observed in the cell lines were not identical to the ones found in primary tumours, they presented major similarities, indicating that these cell lines largely reflect their primary tumour major cytogenetic imbalances.

Comparison of our PDTC and ATC CGH results with previously reported data (Hemmer et al, 1999; Wilkens et al, 2000; Wreesmann et al, 2002; Rodrigues et al, 2004) revealed that all imbalances, described as frequently occurring in the literature, were also observed in the cell lines.

\section{Oligonucleotide microarray analysis}

For oligo-array analysis, only the cell lines were evaluated, owing to lack of well-preserved samples for RNA analysis.

Our work represents the first array expression profile in PDTC. Comparison of the gene expression profiles of the two PDTC 
A
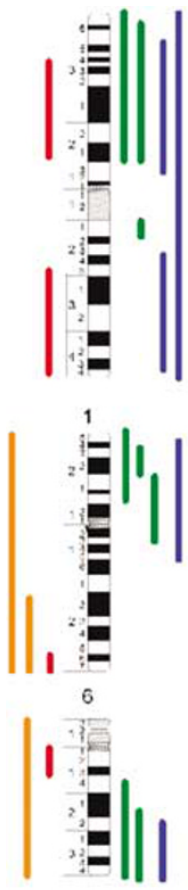

13

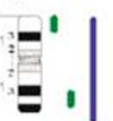

19
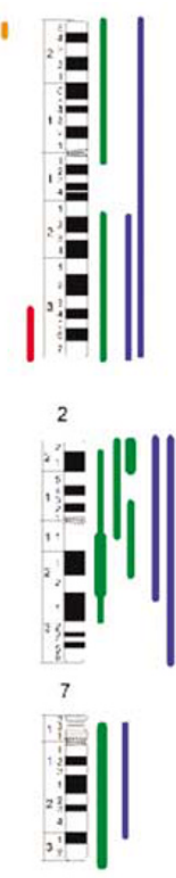

14

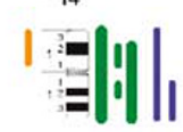

20

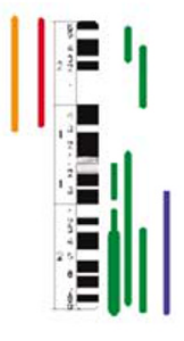

3

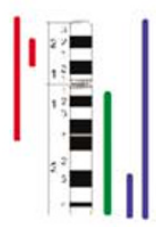<smiles>C1#[I-]C=[V]C1</smiles>

15
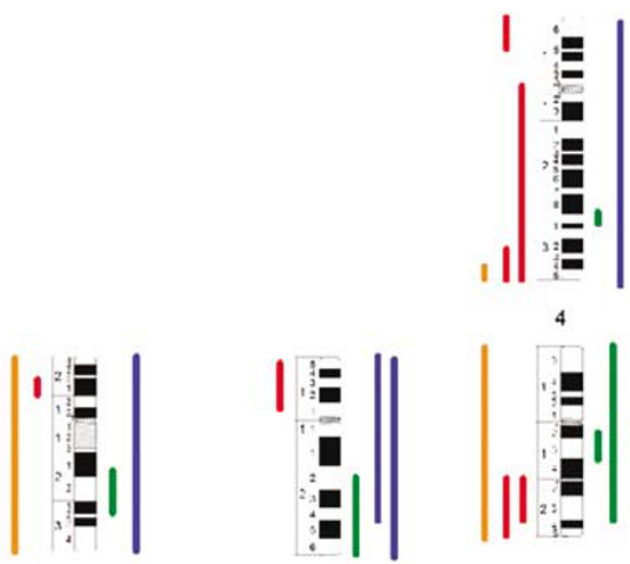

g

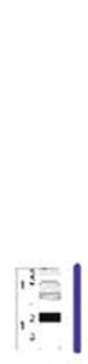

22

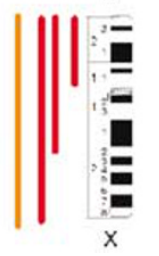

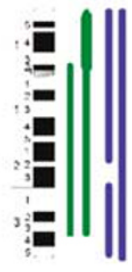

5

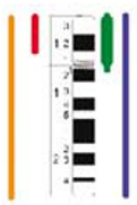

12

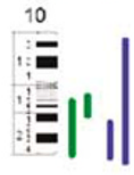

16

17

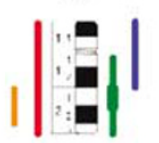

18

B
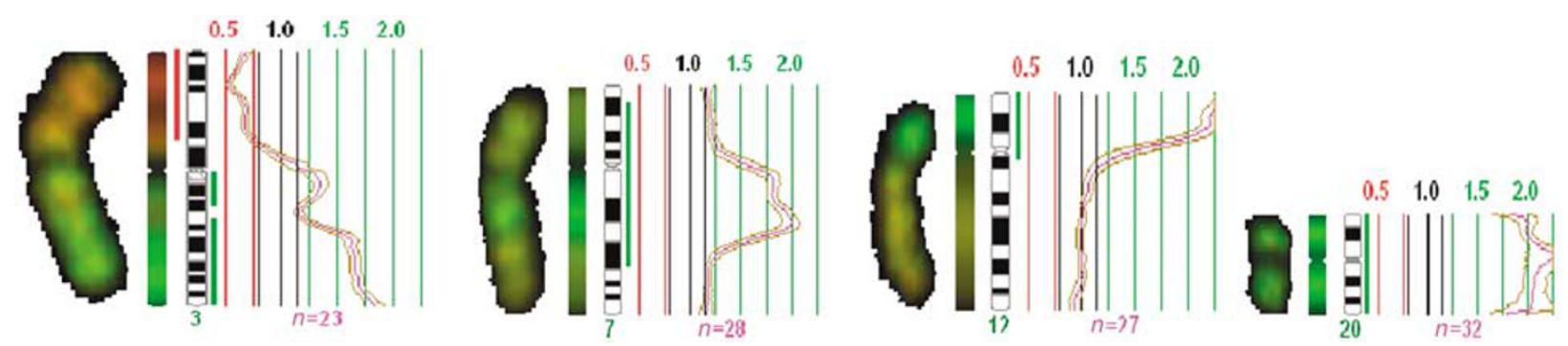

C

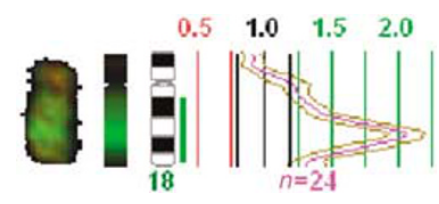

D
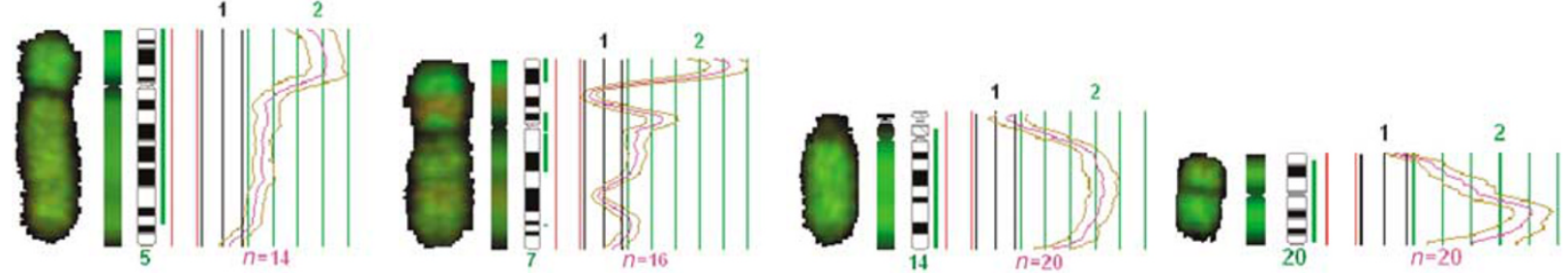

Figure 2 (A) Summary of the DNA copy number changes detected by CGH. Each bar in the left side of the chromosome ideograms represents a loss in one cell line (red-ATC, orange-PDTC) and each bar on the right side of represents a gain in one cell line (green-ATC, blue-PDTC). High-level amplifications are represented by thicker lines. (B) T235 HLA regions at 3q24-qter, 7q। I.2-q22, I2pter-pl I, and 20pter-qter; (C) T238 HLA at I8q21; (D) T24I HLA regions at 5pter-p 12, I4q10-qter and 20p I I.1-qter. 
cancer cell lines with normal thyroid uncovered 4238 significantly $(P<0.001)$ differentially expressed genes. In all, 1079 genes were overexpressed and 3159 were underexpressed (array supplementary data). In Table 2 are depicted the 10 more overexpressed and the 10 more underexpressed genes in PDTC vs normal thyroid. After gene clustering of the differentially expressed genes according to their cellular function, the 10 main categories were in the order: ATP binding, cytoskeleton, cell cycle, mitosis, muscle development, DNA replication, tumour suppressor, transmembrane receptor protein tyrosine kinase signalling pathway, chromosome organisation and biogenesis, and RNA processing (array supplementary data).

When comparing gene expression profiles of the three ATC cell lines with normal thyroid, we found 3367 significantly $(P<0.001)$ differentially expressed genes. In all, 1039 genes were overexpressed and 2328 were underexpressed (array supplementary data).

In Table 2 are depicted the 10 more overexpressed and underexpressed genes in ATC $v s$ normal thyroid. In this group of tumours, gene clustering of the differentially expressed genes according to their cellular function revealed that the most representative were in the order: ATP binding, transport, extracellular space, cell growth and/or maintenance, cell adhesion molecule activity, cytoskeleton, soluble fraction, actin binding, extracellular matrix, and muscle development (array supplementary data). By array analysis we observed in ATC as well as in PDTC that expression downregulation was more relevant than upregulation, whereas by CGH gains were more frequent. A general lack of correlation between chromosomal CGH and array data has previously been described in other neoplasms, namely in colon cancer (Platzer et al, 2002), and may be explained by the recent observations demonstrating that changes in DNA copy number induce changes in the expression of only a limited number of genes. Moreover, the different sensitiveness of the two techniques must also be taken into account.

Comparison between the expression levels at the HLA regions in ATC and normal thyroid revealed that all the differentially expressed genes mapped at these chromosomal regions were overexpressed. In Table 3, we show the set of 40 genes identified to be commonly overexpressed in the three cell lines and mapping at the HLA regions. Of these, 37 represent known genes and three were ORFs (open reading frames).

When comparing the expression profiles of the two types of cell lines, we found 140 significantly differentially expressed genes $(P<0.001)$. Of these, 33 were more expressed in PDTC cell lines and the other 107 were relatively more expressed in the ATC cell lines (array supplementary data). Clustering of these genes according to their cellular function revealed that the most representative group was that of signal transduction molecules followed by groups of genes involved in cell adhesion and motility process (array supplementary data).

Hierarchical clustering using the 140 differentially expressed genes allowed a clear segregation of the tumour cells (Figure $3 \mathrm{~A}$ ). However, we could verify, when selecting only the nine more expressed genes in ATC and in PDTC, that a clear segregation of the two tumour types was still observable both my hierarchical clustering (Figure 3B) and PCA analysis (Figure 3C). All these molecules are known to be involved in cancer process, some being identified as tumour suppressor genes (e.g. GPR54 and CLDN23) (Kotani et al, 2001; Katoh and Katoh, 2003) and others related to angiogenesis (e.g. CXL1 and EDNRA) (Hosoda et al, 1992; Shintani et al, 2004) or cell migration capacities (e.g. PLAU) (Guo et al, 2002).

\section{Quantitative real-time PCR}

Regarding the genes mapped at the HLA regions, TaqMan ${ }^{\circledR}$ PCR experiments confirmed the overexpression noted in microarrays
Table 2 List of the 10 more up- and downregulated genes in the comparisons PDTC/normal thyroid and ATC/normal thyroid

\begin{tabular}{lc} 
Gene & LBFC \\
\hline The I 0 genes more upregulated in the comparison PDTC/normal thyroid & \\
T-LAK cell-originated protein kinase (PBK) & 66.95 \\
Prostate differentiation factor (GDFI5) & 43.56 \\
Anillin, actin-binding protein (scraps homolog, Drosophila) (ANLN) & 39.80 \\
Leucine zipper protein FKSG I 4 (FKSG I 4) & 31.82 \\
Hyaluronan-mediated motility receptor (RHAMM) (HMMR) & 28.71 \\
Activin $\beta$ E (INHBE) & 27.29 \\
Discs, large homolog 7 (Drosophila) (DLG7) & 24.57 \\
Thymidylate synthetase (TYMS) & 24.14 \\
Topoisomerase (DNA) II $\alpha$ I 70 kDa (TOP2A) & 23.84 \\
Asp (abnormal spindle)-like, microcephaly associated & 23.64 \\
(Drosophila) (ASPM) &
\end{tabular}

The 10 genes more downregulated in the comparison PDTC/normal thyroid Lumican (LUM)

Mucin 7, salivary (MUC7)

Solute carrier family 26, member 4 (SLC26A4)

$-152$

Histatin 3 (HTN3)

Histatin | (HTN I)

Integral membrane protein 2A (ITM2A)

Statherin (STATH)

Amylase, $\alpha$ 2A; pancreatic (AMY2A)

SI00 calcium-binding protein A8 (calgranulin A) (S100A8)

Homo sapiens-transcribed sequence with weak similarity to protein ref:NP_060312.I (H. sapiens) hypothetical protein FLJ20489 [Homo sapiens]

The 10 genes more upregulated in the comparison ATC/normal thyroid Homo sapiens-transcribed sequences

Phorbol-|2-myristate- |3-acetate-induced protein | (PMAIPI)

Fibronectin I (FNI)

Melanoma antigen, family A, 6 (MAGEA6)

$\mathrm{LI}$ cell adhesion molecule (hydrocephalus, stenosis of aqueduct

of Sylvius I, MASA (mental retardation, aphasia, shuffling gait and adducted thumbs) syndrome, spastic paraplegia I) (LICAM)

Gb:AF043337.I /DB_XREF = gi: I 264 I $914 / \mathrm{GEN}=$ IL8 /

$\mathrm{FEA}=\mathrm{FLmRNA} / \mathrm{CNT}=1 / \mathrm{TID}=\mathrm{Hs} .624 .1 / \mathrm{TIER}=\mathrm{FL} / \mathrm{STK}=0$

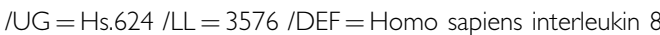

C-terminal variant (IL8) mRNA, complete cds. /

$\mathrm{PROD}=$ interleukin 8 C-terminal variant $/ F L=g b: A F 043337$.

E2F transcription factor 7 (E2F7)

Human full-length cDNA $5^{\prime}$-end of clone CSODK007YB08 of

$-133.1 \mid$

$-132.0$

$-122.6$

$-107.75$

$-98.39$

$-93$

$-88.3$

$-79.35$

HeLa cells of Homo sapiens (human)

Melanoma antigen, family A, 3 (MAGEA3)

Anillin, actin-binding protein (scraps homolog, Drosophila) (ANLN)

54.26

46.41

41.06

31.36

30.98

30.82

The 10 genes more downregulated in the comparison ATC/normal thyroid Histatin 3 (HTN3)

Solute carrier family 26, member 7 (SLC26A7)

Histatin I (HTN I)

Mucin 7, salivary (MUC7)

Glycine amidinotransferase (L-arginine:glycine

amidinotransferase) (GATM)

Solute carrier family 26, member 4 (SLC26A4)

$\alpha$ and $\mu$ polypeptides $(\mid G)$

Thyroglobulin (TG)

Thyroid peroxidase (TPO)

Integral membrane protein 2A (ITM2A)

PDTC $=$ poorly differentiated thyroid carcinomas. ATC $=$ anaplastic thyroid carcinomas. $\mathrm{LBFC}=$ lower bound of fold change.

analysis for all chosen genes: ARNTL2, AURKA, CLDN1, PMAIP1, and $R B A K$. Of remark is that the elevated expression values of these genes were also observed in the three additional ATC primary samples. As for $C D H 13$ and PLAU, TaqMan ${ }^{\circledR}$ PCR 
Table 3 Set of genes found to be commonly differentially expressed in the comparison of the three ATC cell lines to normal thyroid

LBFC in

Genes 3ATC/NTHY

\#3q Claudin I (CLDN I)

Butyrylcholinesterase $(B C H E)$

SMC4 structural maintenance of chromosomes 4-like I

(SMC4LI)

Epithelial cell transforming sequence 2 oncogene (ECT2)

Transferrin receptor ( $p 90, C D 71)$

Karyopherin $\alpha 4$ (importin $\alpha 3$ ) (KPNA4)

BM-OI I protein (BM-OII)

Likely ortholog of mouse IRAI protein (IRAI)

\#5

FLJ32363 protein (FLJ32363)

\#7

Activator of S-phase kinase (ASK)

Zinc finger protein 92 (HTFI 2)

FLJ20073 protein (FLJ20073)

Asparagine synthetase (ASNS)

$\mathrm{RB}$-associated $\mathrm{KRAB}$ repressor (RBAK)

Hypothetical protein FLJ20097 (FLJ20097)

Origin recognition complex, subunit 5-like (yeast) (ORCL5)

Paternally expressed 10 (PEG/O)

$\# 12$

RAD5 I -interacting protein (RAD5 | AP I)

Forkhead box MI (FOXMI)

Aryl hydrocarbon receptor nuclear translocator-like 2 (ARNTL2)

\#14

KIAA/ 333 (KIAA/333)

Suppressor of Ty 16 homolog (S. cerevisiae) (SUPTI 6 H)

Mitogen-activated protein kinase kinase kinase kinase 5 (ASKI)

WD repeat and HMG-box DNA-binding protein I (WDHDI)

\#18

Phorbol- | 2-myristate- | 3-acetate-induced protein I (PMAIPI)

\#20

Ubiquitin-conjugating enzyme E2C (UBEC2C)

Synaptosomal-associated protein, $25 \mathrm{kDa}$ (SNAP25)

Chromosome 20 open reading frame 97 (C20ORF97)

Aurora kinase A (AURKA)

Eukaryotic translation initiation factor 2 , subunit $2 \beta, 38 \mathrm{kDa}$

(EIF2S2)

Syntaxin 16 (STX16)

Chromosome 20 open reading frame 6 (C200RF6)

CGI-09 protein (CGl-09)

TPX2, microtubule-associated protein homolog (Xenopus laevis)

(TPX2)

Chromosome 20 open reading frame 100 (C20ORFI00)

Phospholipase C, $\beta$ । (phosphoinositide-specific) (PLC beta)

CSEI chromosome segregation I-like (yeast) (CSEI)

Solute carrier organic anion transporter family, member 4AI

(SLCO4Al)

Transglutaminase 2 (C polypeptide, protein-glutamine-

$\gamma$-glutamyltransferase) (TGM2)

MCM8 minichromosome maintenance deficient 8 (S. cerevisiae) (MCM8)

ATC = anaplastic thyroid carcinomas; HLA = high-level amplification regions; $\mathrm{LBFC}=$ lower bound of fold change.

experiments also confirmed array results (Figure 4). MannWhitney statistical testing of the results revealed significant differences between the gene expression levels in the two types of neoplasms. The obtained $P$-values for $C D H 13$ was $=0.0275$ and for PlAU was $=0.0377$.

\section{DISCUSSION}

Alterations in the structure or expression of proto-oncogenes and tumour suppressor genes were determined to be fundamental to cancer development and progression by respectively up- and downregulating cellular control pathways (Vogelstein and Kinzler, 1998). In thyroid the identification of these genes and how their alterations contribute to the pathogenesis of the two most aggressive cancer forms: poorly differentiated and anaplastic carcinomas are still ill defined. The use of cancer cell lines has represented an invaluable tool for gene discovery in other tumour types. As such, and to obtain information about the altered genes other than those already known to be associated to these two tumour types, we performed an evaluation at the DNA and RNA levels using cytogenetic and oligo-array analysis in five new cell lines established from two PDTC and three ATC.

Poorly differentiated thyroid carcinomas are classified according to the WHO as neoplasms presenting morphological and clinical criteria in between well-differentiated thyroid: papillary and follicular carcinomas, and anaplastic cancers.

Chromosomal translocations, inversions and duplications are the known mechanisms of oncogene activation in cancer. At the cytogenetic level, the most frequent structural rearrangements in papillary cancers are abnormalities with breakpoints at $10 \mathrm{q} 11.2$ (Roque et al, 2001). These alterations lead to the activation of the $R E T$ proto-oncogene. In our PDTC cell lines, although papillary features were still recognised, none of them presented alterations with breakpoints at 10q11.2 (RET gene was also observed not to be recombined - data not shown). However, of remark is the observation that by array analysis the most significantly overexpressed gene was $P B K$. The protein encoded by $P B K$ is a serine/ threonine kinase which belongs to the MAPK kinase protein family (Abe et al, 2000). The involvement of $P B K$ has not previously been observed in thyroid cancer, but previous data (Mellilo et al, 2005) have demonstrated that the constitutive activation of effectors along the $M A P K / E R K$ signalling pathway display a key role in the genesis and progression of a substantial proportion of papillary tumours. Our array findings thus reinforce the importance of this signalling pathway in the tumourigenesis of thyroid carcinomas with papillary characteristics.

P53 is the only tumour suppressor gene identified to be involved in the pathogenesis of PDTC. Molecular studies revealed, however, that the frequency of tumours with mutations at this gene is low: $\sim 25 \%$ of cases (Wynford-Thomas, 1997). In our study, we were able to identify a group of other tumour suppressor genes which were downregulated in these carcinomas, and may have a key role in their development or progression. PLAGL1 gene, which maps at 6q24.2, and $C D H 1$ mapping at 16q22.1, were those with the highest LBFC, respectively, -33.87 and -7.06 . The involvement of PLAGL1 has not been previously noted in PDTC. At variance, participation of $C D H 1$, which codes for E-cadherin in thyroid tumorigenesis has been described. Rocha et al (2003) studied 17 cases of primary PDTC and showed that loss of E-cadherin was a common feature of these tumours.

In our array analysis, in the list of the 10 more underexpressed genes in ATC, we found thyroglobulin and thyroid peroxidase. This finding is in keeping with a preceding array study by Onda et al (2004) in a group of 11 ATC cell lines, and confirms previous studies demonstrating that in anaplastic tumours there is loss of the most characteristic thyroid cellular function, that is, synthesis of T4 and T3 hormones (Elisei et al, 1994). Known tumour suppressor genes, although not included in this list, were noted as downregulated in the array analysis for example, the FHIT $(\mathrm{LBFC}=-5.39)$ at $3 \mathrm{p} 14.2 ; S T 7(\mathrm{LBFC}=-2.95)$ at $7 \mathrm{q} 31$ or $C L D N 23$ $(\mathrm{LBFC}=-4.58)$ at $8 \mathrm{p} 23.1$. Our results strengthen $\mathrm{LOH}$ studies (Kitamura et al, 2001) carried out in ATC that showed frequent allele loss at multiple chromosomal sites in this carcinoma type. Specifically interesting is the underexpression of the ST7 tumour 
A

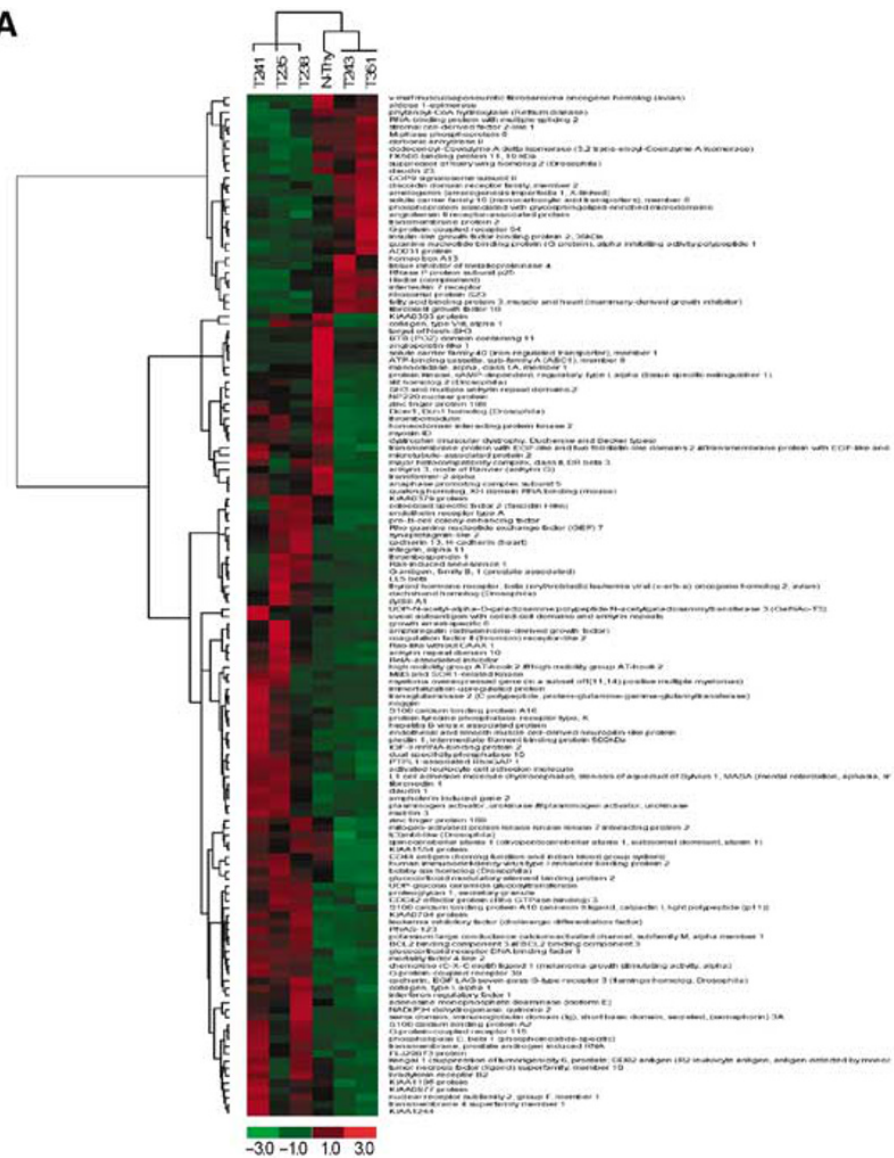

B

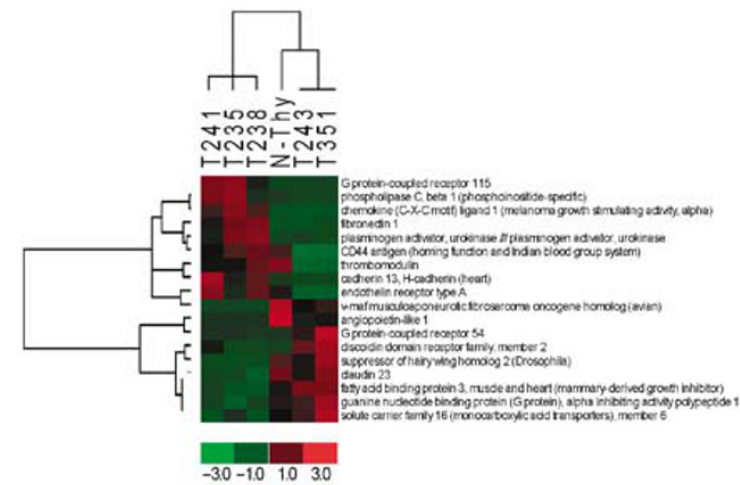

C

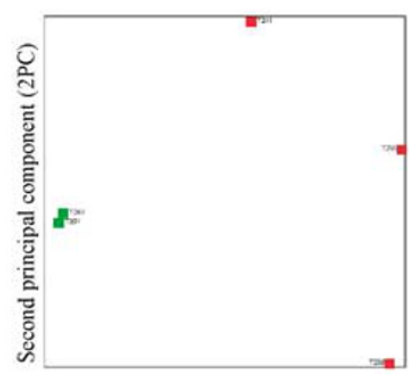

First principal component (IPC)

Figure 3 (A) Hierarchical clustering of the genes and samples using the I 40 differentially expressed genes between the ATC and PDTC. Hierarchical clustering (B) and PCA analysis $(\mathbf{C})$ of the genes and samples using the 18 genes that allowed a distinction between ATC and PDTC.

suppressor gene (Zenklusen et al, 2001) at 7q31, a region that has been reported as frequently deleted in ATC also by previous CGH studies (Rodrigues et al, 2004).

Gene amplification was observed to be a common phenomenon in ATC (Roque et al, 1998). In this study we were able to define for the first time the nature of the amplicon target genes present in this type of carcinomas. We found a set of 40 commonly overexpressed genes at the HLA (Table 3), which included molecules that are known to be involved in the regulation of centrosome duplication, chromosome orientation, and segregation process in cells such as: AURKA, TPX2, CSE-1, MCM8 or SMC4 (Adams et al, 2001). Genes related to transcription regulation (e.g. $R B A K$ at $7 \mathrm{p} 22.1$ and $A R N T L$ at $12 \mathrm{p}$ ) and to cell adhesion process like CLDN1. Noteworthy was the observation of PMAIP1 as an amplicon target at 18q21.32. PMAIP1 is a gene expressed in cells in response to hypoxia, allowing cell adaptation to the altered metabolic demands induced by decreased oxygen levels (Yakovlev et al, 2004). Kim et al (2004) demonstrated that PMAIP1 promoter directly responds to hypoxia via the hypoxia-induced factor (HIF1A). The $\beta$-subunit of HIF1A is ARNT. Of remark is the fact that ARNTL2 was one of the overexpressed genes located at 12p11.3. Anaplastic thyroid carcinomas invade, infiltrate and rapidly grow in the surrounding tissues. In this context, we suggest that during the amplification process autocrine lopps are created that may confer important growth advantages to the neoplasms. The results obtained by TaqMan ${ }^{\circledR}$ PCR for PMAIP1 and ARNTL2, but also for AURKA, CLDN1, and RBAK demonstrated that the amplification process of these genes is not only cell line specific, but also occurs in primary ATC. Therefore reinforcing the importance of these molecules in thyroid anaplastic characteristics.
Anaplastic thyroid carcinomas and PDTC have distinct disease courses. The former have a dismal prognosis, whereas in PDTC the median survival rate is 3-5 years (DeLellis and Williams, 2004). Assuming that these biologic different behaviours are a reflection of the deregulation of distinct molecular characteristics in each tumour type, we addressed this hypothesis by comparing the ATC and PDTC cell line expression profiles. We found that the main molecular differences between them were related to signal transduction pathways, cell adhesion and motility process, and this involved 140 genes.

Major advances were made in the treatment of chronic myeloid leukaemias and gastrointestinal tumours by using drugs that directly inhibit the pathways deregulated by the BCL2-ABL fusion gene and c-KIT mutations (Hochhaus, 2004). These genetic alterations are usually the only aberrations in these neoplasms at the time of diagnosis and treatment is in fact most of the times effective. Neoplasias such as PDTC or ATC are at variance characterised by a profound genomic instability and the currently used therapies especially in ATC cases are useless. In our work, hierarchical and PCA analysis allowed to verify that a group of 18 cancer genes served as a basis to differentially characterise anaplastic and poorly differentiated thyroid carcinomas. TaqMan ${ }^{\circledR}$ PCR experiments performed for two of these molecules, $C D H 13$ and PLAU, corroborated array results revealing significant expression levels differences between the two cancer types, not only in the cell lines, but also in an additional set of primary 14 PDTC and three ATC. Overall, our findings point out that the molecular pathways of ARNTL2, AURKA, CDH13, CLDN1, $P M A I P 1, P L A U$, and $R B A K$ should be further investigated order to understand the mechanisms by which these genes may be linked 


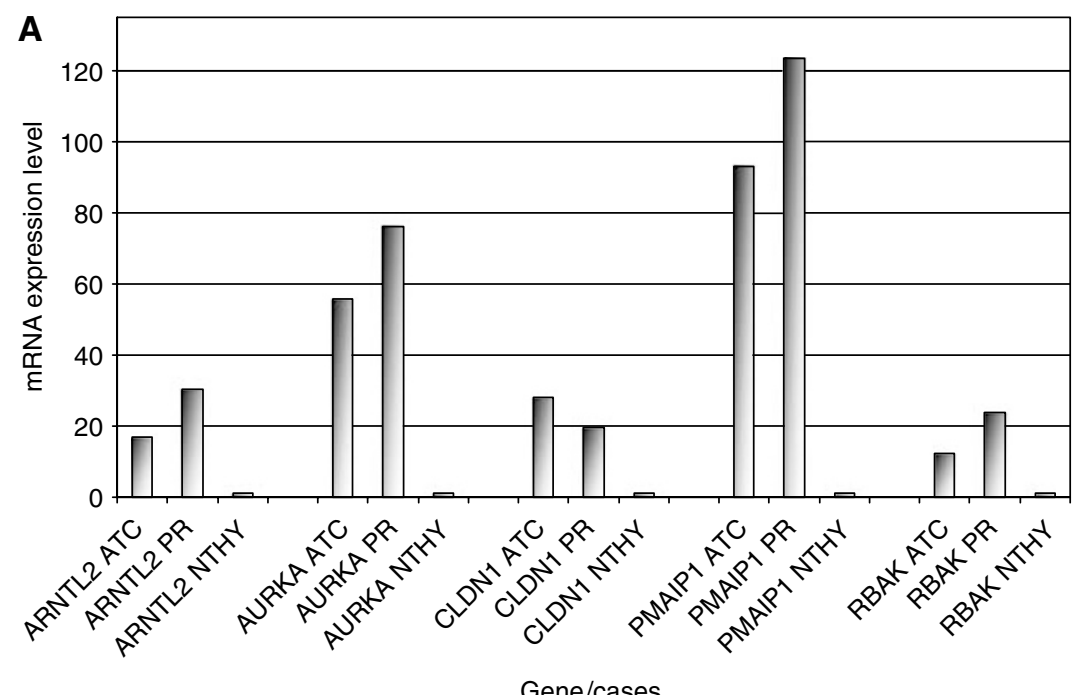

Gene/cases

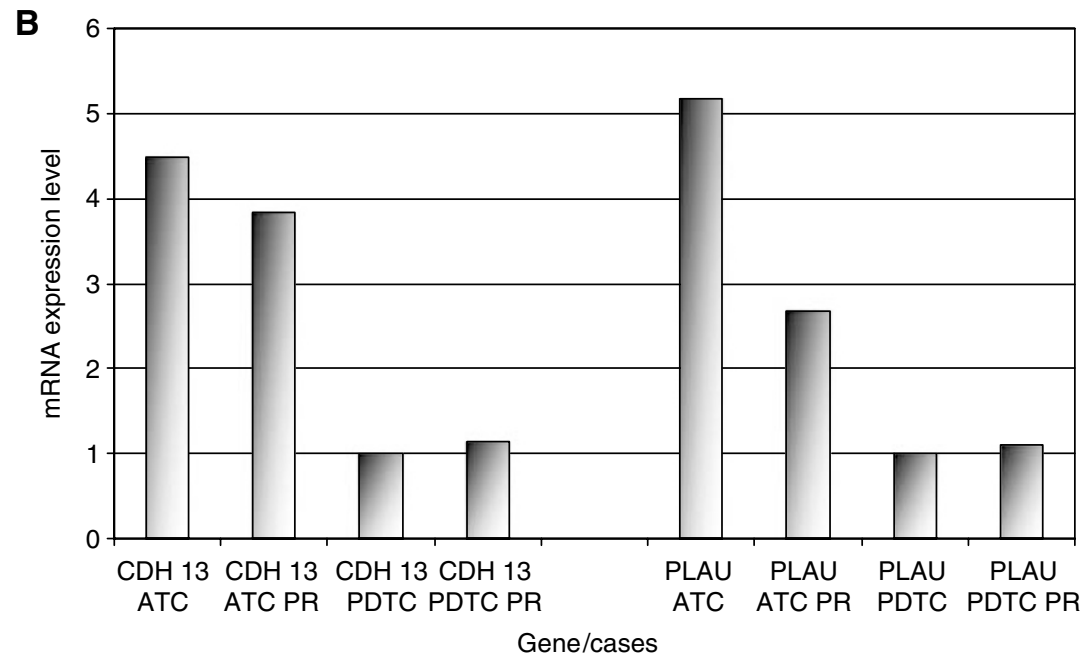

Figure 4 Real-time quantitative PCR/TaqMan results (measured by gene/GAPDH). The results were scaled by dividing each set of results by the minor value within the set. (A) Validation for five of the overexpressed genes within the amplicons, according to microarray analysis: ARNTL2, AURKA, CLDN I, PMAIPI, and RBAK. For each gene; first column - averages of the results obtained for all the analysed ATC cases (three cell lines + three primary cases); second column - averages for the three primary ATC; third column — results for normal thyroid. (B) Validation for two of the genes where expression was distinct in PDTC vs ATC, by array results: CDHI 3 and PLAU. For each gene: first column-averages of the results obtained for all ATC analysed cases (three cell lines + three primary cases); second column—averages for the three primary ATC's; third column-averages of the results obtained for all PDTC analysed cases (two cell lines + I4 primary cases); fourth column—averages for all the I4 primary PDTC.

to anaplastic therapeutic resistance and to design concerted therapies that could improve ATC prognosis.

\section{ACKNOWLEDGEMENTS}

We thank Dr Ana Paula Elias, Cristina Casalou, and Sara Vieira for their assistance in the experiments, Dr Ana Luisa Catarino and Teresa Pereira from the Pathology Department of the Portuguese
Cancer Institute for kindly acquiring the histological pictures of the tumors, Dr Sérgio Dias for all the support at the Laboratory for the fulfilment of this work, and Professor Sérgio Castedo for the helpful critical review of this paper.

Supplementary Information accompanies the paper on British Journal of Cancer website (http://www.nature.com/bjc)

\section{REFERENCES}

Abe Y, Matsumuto S, Kito K, Ueda N (2000) Cloning and expression of a novel MAPKK-like protein kinase, lymphokine-activated killer T-celloriginated protein kinase, specifically expressed in the testis and activated lymphoid cells. J Biol Chem 275: 21525-21531
Adams RR, Carmena M, Earnshaw WC (2001) Chromosomal passengers and the (aurora) ABCs of mitosis. Trends Cell Biol 11: 49-54

Asakawa H, Kobayashi T, Komoike Y, Yanagawa T, Takahashi M, Wakasugi E, Maruyama H, Tamaki Y, Matsuzawa Y, Moden M (1996) 
Establishment of anaplastic thyroid carcinoma cell lines useful for analysis of chemosensivity and cancerigenesis. J Clin Endocrinol Metab 81: $3547-3552$

Brodeur GM (2003) Neuroblastoma: biological insights into a clinical enigma. Nat Rev Cancer 3: 203-216

DeLellis RA, Williams ED (2004) Tumors of the thyroid and parathyroid. In World Health Organization Classification of Tumors. Pathology and Genetics of Tumors of Endocrine Organs DeLellis RA, Lloyd RV, Heitz PU, Eng C (eds) pp 49-93. Lyon: IARC Press

Elisei R, Pinchera A, Romei C, Gryczynska M, Pohl V, Maenhaut C, Fugazzola L, Pacini F (1994) Expression of thyrotropin receptor (TSH-R), thyroglobulin, thyroperoxidase, and calcitonin messenger ribonucleic acids in thyroid carcinomas: evidence of TSH-R gene transcript in medullary histotype. J Clin Endocrinol Metab 78: 867-871

Garcia-Rostan G, Tallini G, Herrero A, D’Aquila TG, Carcangiu ML, Rimm DL (1999) Frequent mutation and nuclear localization of $\beta$-catenin in anaplastic thyroid carcinoma. Cancer Res 59: 1811 - 1815

Guo Y, Mazar AP, Lebrun JJ, Rabbani SA (2002) An antiangiogenic urokinasederived peptide combined with tamoxifen decreases tumor growth and metastasis in a syngeneic model of breast cancer. Cancer Res 62: $4678-4684$

Hemmer S, Wasenius VM, Knuutila S, Franssila K, Joensuu H (1999) DNA copy number changes in thyroid carcinoma. Am J Pathol 154: $1539-1547$

Hochhaus A (2004) Imabitinib mesylate (Glivec, Gleevec) in the treatment of chronic myelogenous leukaemia (CML) and gastrointestinal stromal tumors (GIST). Ann Hematol 83(Suppl 1): S65-S66

Hosoda K, Nakao K, Tamura N, Arai H, Ogawa Y, Suga S, Nakanishi S, Imura H (1992) Organization, structure, chromosomal assignment, and expression of the gene encoding the human endothelin-A receptor. J Biol Chem 26: $18797-18804$

Katoh M, Katoh M (2003) CLDN23 gene, frequently down-regulated in intestinal-type gastric cancer, is a novel member of CLAUDIN gene family. Int J Mol Med 11: 683-689

Kim JY, Ahn HJ, Ryu JH, Suk K, Park JH (2004) BH3-only protein Noxa is a mediator of hypoxic cell death induced by hypoxia-inducible factor 1 alpha. J Exp Med 199: 113-124

Kitamura Y, Shimizu K, Ito K, Tanaka S, Emi M (2001) Allelotyping of follicular thyroid carcinoma: frequent allelic losses in chromosome arms 7q, 11p, and 22q. J Clin Endocrinol Metab 86: 4268-4272

Kotani M, Detheux M, Vandenbogaerde A, Communi D, Vanderwinden JM, Le Poul E, Brezillon S, Tyldesley R, Suarez-Huerta N, Vandeput F, Blanpain C, Schiffmann SN, Vassart G, Parmentier M (2001) The metastasis suppressor gene KiSS-1 encodes kisspeptins, the natural ligands of the orphan G protein- coupled receptor GPR54. J Biol Chem 276: $34631-34636$

Lacroix L, Lazar V, Michiels S, Ripoche H, Dessen P, Talbot M, Caillou B, Levillain JP, Schlumberger M, Bidart JM (2005) Follicular thyroid tumors with the PAX8-PPAR $\gamma 1$ rearrangement display characteristic genetic alterations. Am J Pathol 167: 223-231

Mellilo RM, Castellone MD, Guarino V, De Falco V, Cirafici AM, Salvatore G, Caiazzo F, Basolo F, Giannini R, Kruhoffer M, Orntoft T, Fusco A, Santoro M (2005) The RET/PTC-RAS-BRAF linear signaling cascade mediates the motile and mitogenic phenothype of thyroid cancer cells. J Clin Invest 115: $1068-1081$

Mitelman F (1995) ISCN. An Iternational System for Human Cytogenetic Nomenclature. Switzerland: Karger Landers, Basel.

Mizutani K, Onda M, Asaka S, Akaishi J, Miyamoto S, Yoshida A, Nagahama M, Ito K, Emi M (2005) Overexpressed in anaplastic thyroid carcinoma-1 (OEATC-1) as a novel gene responsible for anaplastic thyroid carcinoma. Cancer 103: $1785-1790$

Onda M, Emi M, Yoshida A, Miyamoto S, Akaishi J, Asaka S, Mizutani K, Shimizu K, Nagahama M, Ito K, Tanaka T, Tsunoda T (2004) Comprehensive gene expression profiling of anaplastic thyroid cancers with cDNA microarrays of 25344 genes. Endocr-Relat Cancer 11: $843-854$

Platzer P, Upender MB, Wilson K, Willis J, Lutterbaugh J, Nosrati A, Willson Jk, Mack D, Ried T, Markowitz S (2002) Silence of chromosomal amplifications in colon cancer. Cancer Res 62: 1134-1138

Ringel MD, Greenberg M, Chen X, Hayre N, Suzuki K, Priebat D, Saji M, Burman KD (2000) Cytotoxic activity of 2',2'- difluorodeoxycytine (gemcitabine) in poorly differentiated thyroid carcinoma cells. Thyroid 10: $865-869$

Rocha AS, Soares P, Fonseca E, Cameselle-Teijeiro J, Oliveira MC, Sobrinho-Simões M (2003) E-cadherin loss rather than beta-catenin alterations is a common feature of poorly differentiated thyroid carcinomas. Histopathology 42: $580-587$

Rodrigues RF, Roque L, Rosa-Santos J, Cid O, Soares J (2004) Chromosomal imbalances associated with anaplastic transformation of follicular thyroid carcinomas. Br J Cancer 90: 492-496

Roque L, Soares J, Castedo S (1998) Cytogenetic and fluorescence in situ hybridization studies in a case of anaplastic thyroid carinoma. Cancer Genet Cytogenet 103: 7-10

Roque L, Nunes VM, Ribeiro C, Martins C, Soares J (2001) Karyotypic characterization of papillary thyroid carcinomas. Cancer 92: $2529-2538$

Rosai J, Carcangiu ML, DeLellis R (1992) Tumors of the Thyroid Gland. Atlas of Tumor Pathology 3rd Series. Washington, DC: Armed Forces Institute of Pathology.

Shintani S, Ishikawa T, Nonaka T, Li C, Nakashiro K, Wong DT, Hamakawa $\mathrm{H}$ (2004) Growth-regulated oncogene-1 expression is associated with angiogenesis and lymph node metastasis in human oral cancer. Oncology 66: $316-322$

Sorrentino R, Libertini S, Pallante PL, Troncone G, Palombini L, Bavetsias V, Spalletti-Cernia D, Laccetti P, Linardopoulos S, Chieffi P, Fusco A, Portella G (2005) Aurora B overexpression associates with the thyroid carcinoma undifferentiated phenotype and is required for thyroid carcinoma cell proliferation. J Clin Endocrinol Metab 90: 928-935

Vogelstein B, Kinzler KW (1998) The Genetic Basis of Human Cancer. USA: McGraw-Hill Companies Inc.

Wilkens L, Benten D, Tchinda J, Brabant G, Potter E, Dralle H, von Wasielewski R (2000) Aberrations of chromosome 5 and 8 as recurrent cytogenetic events in anaplastic carcinoma of the thyroid as detected by fluorescence in situ hybridization and comparative genomic hybridization. Virchows Arch 436: 312-318

Wreesmann VB, Ghossein RA, Patel, SG, Harris CP, Schnaser EA, Shaha AR, Tuttle RM, Shah JP, Rao, PH, Singh B (2002) Genome-wide appraisal of thyroid cancer progression. Am J Pathol 161: 1549-1556

Wynford-Thomas D (1997) Origin and progression of thyroid epithelial tumours: cellular and molecular mechanisms. Horm Res 47: 145-157

Yakovlev AG, Di Giovanni S, Wang G, Liu W, Stoica B, Faden AI (2004) BOK and NOXA are essential mediators of p53-dependent apoptosis. J Biol Chem 279: $28367-28374$

Zenklusen JC, Conti CJ, Green ED (2001) Mutational and functional analyses reveal that ST7 is a highly conserved tumor-suppressor gene on human chromosome 7q31. Nat Genet 27: 392-398 